\title{
Cinema documentário, pesquisa e método Desafios para os estudos interdisciplinares
}

\author{
Natália Ramos', José Francisco Serafim²
}

\begin{abstract}
Resumo
Destaca-se neste texto a importância, caracteristicas e vantagens da metodologia filmica na pesquisa e na interdisciplinaridade. Salientam-se igualmente alguns principios e procedimentos metodológicos, técnicos e analiticos sobre a utilização da imagem documental na pesquisa. Após um rápido panorama histórico e metodológico sobre o filme documentário, desde o surgimento do cimematógrafo em 1895 na França, passando por dois de seus maiores representantes, Robert Flaherty e Dziga Vertov, busca-se mostrar o interesse deste aporte metodológico. Este inclui a imagem estática e em movimento nos trabalhos acadêmicos e foi iniciado pelos pesquisadores Margaret Mead e Gregory Bateson nos anos 1930 , seguidos por pesquisadores franceses tais que Marcel Griaule e Jean Rouch, nos anos 1930 e 1940, vindo a se tornar uma disciplina, no sentido amplo do termo, quando a pesquisadora Claudine de France publica o texto Cinema e Antropologia no final do anos 1970. A partir deste momento esta nova disciplina a antropologia filmica começa a ser um instrumental valioso para a compreensão do Homem e das atividades humanas em situação de pesquisa.
\end{abstract}

Palavras chaves: Cinema documentário; pesquisa; metodologia filmica

\begin{abstract}
This text focuses on the importance, characteristics and advantages of the filmic methodology in research and in interdisciplinary studies. It points out too some principles and methodological behaviours, techniques and analytics about the use of the documentary image in the research. Initially, there is a short historical and methodological review on documentary film, from the beginning of the cimematograph in 1895 in France, going through two of its major representatives - Robert Flaherty and Dziga Vertov. Then, we demonstrate the interest for methodological approach which includes still image and in movement in academic works. This methodology, started by the researchers Margaret Mead and Gregory Bateson in the 1930s, followed by the French Jean Rouch in the 1940s, became a field of study on its own when researcher Claudine de France published her text Cinema and Anthropology, at the end of the 1970s. From then onwards, this new discipline - filmic anthropology - became a precious tool for the understanding of Man and human activities in research situation.
\end{abstract}

Keywords: Documentary film, research; filmic methodology

\footnotetext{
' Natália Ramos doutorado em Psicologia Social realizado na Universidade de Paris V. Professora e pesquisadora na Universidade Aberta de Lisboa e diretora do Mestrado em Comunicação e Saúde

2 José Francisco Serafim realizou o doutorado em Cinema Documentário na Universidade Paris X-Nanterre. É atualmente professor e pesquisador na Faculdade de Comunicação e no Programa de Pós-Graduação em Comunicação e Cultura Contemporâneas da Universidade Federal da Bahia
} 


\section{Introdução}

A imagem tem vindo a ocupar um lugar privilegiado na pesquisa, no âmbito das diferentes áreas disciplinares, em particular, das Ciências Humanas e Sociais.A pesquisa envolvendo a imagem estática ou em movimento pode reportar-se à observação e análise da imagem relativa ao processo filmado por outros ou ao processo de registro e edição das imagens coletadas pelo próprio pesquisador.

É nesta segunda perspectiva que neste artigo abordaremos algumas questões metodológicas relativas à utilização do cinema, particularmente do documentário, para a observação e conhecimento do Homem, na sua unidade e diversidade e nos diferentes contextos sociais e culturais.

Sublinha-se a importância do filme para desvendar aspectos da sociedade por vezes à margem, difusos ou ostensivos a fim de trazê-los para o campo do visível e partilha-los, não só, com as pessoas filmadas, mas também com outros pesquisadores e a comunidade em geral, indo ao encontro da perspectiva de "antropologia partilhada" defendida pelo antropólogo-cineasta Jean Rouch (1979).

Os métodos e as técnicas audiovisuais trouxeram mudanças ao nível dos paradigmas conceituais, teóricos e práticos abrindo novas perspectivas de pesquisa e possibilitando a análise da comunicação em suas diferentes dimensões.

Ao nível metodológico, a imagem animada sonora ao integrar a comunicação verbal e não verbal e os contextos onde se desenrolam as atividades, permite uma abordagem holística, interacionista e apreender a "situação total", utilizando a terminologia dos antropólogos Marcel Mauss (1934) e Margaret Mead (1951, 1979)

Apresentam-se neste artigo algumas características e especificidades da utilização do método filmico na antropologia filmica e na pesquisa.

\section{Contextualização teórica e metodológica}

Na França, os irmãos Louis e Auguste Lumière, foram não só os inventores do cinema em 1895 com a criação do "cinematógrafo", como também os pioneiros e precursores do cinema documentário, 
filmando diversas cenas da vida familiar e quotidiana que projetaram em pequenos filmes no Salão Índio do Grand Café de Paris.

Alguns meses depois da primeira sessão pública dos filmes dos irmãos Lumière, ocorrida em 28 de dezembro de 1895, é igualmente na França que o médico e etnólogo Felix-Louis Regnault (1896), será o primeiro pesquisador a sublinhar o interesse do filme com objetivo de pesquisa, sobretudo para o estudo das técnicas corporais, do movimento e posturas em diferentes grupos étnicos e ainda para observar fatos que escapam aos nossos sentidos porque demasiados rápidos e fugazes. Para este pesquisador " $O$ cinema aumenta a nossa visão no tempo como o microscópio a aumentou no espaço. Ele nos permite observar fatos que escapam aos nossos sentidos porque demasiados rápidos e fugazes".

Em 1898, na Inglaterra o pesquisador Alfred C. Haddon será o pioneiro ao introduzir o instrumental filmico na pesquisa multidisciplinar no Estreito de Torres (situado entre a Austrália e a Nova Guiné) para estudar diversos aspectos sociais, culturais e rituais de algumas populações locais desta região do Pacífico e captar diversas manifestações possíveis de serem registradas através da imagem em movimento.

O cinema documentário começará a ganhar importância nos anos 1920, com os dois grandes pioneiros do gênero, o norte-americano Robert Flaherty e o russo Dziga Vertov. Com efeito, Nanook, do Norte, realizado em 1922 por Flaherty e $O$ homem com a câmera dirigido por Vertov em 1920, constituem dois modelos do cinema documentário, os quais irão marcar a trajetória deste gênero cinematográfico.

Flaherty, no seu documentário Nanook, do Norte, vai observar e filmar a vida quotidiana de uma família Inuit no norte do Canadá, ao longo das estações do ano, sublinhando a vida harmoniosa deste grupo, apesar da luta pela sobrevivência em uma região inóspita e em condições adversas. A temática da ralação entre o Homem e a natureza será o fio condutor dos trabalhos posteriores realizados por este cineasta, tais como Moana (1926) realizado nas ilhas Samoa e O homem de Aran (1934) filmado na costa oeste irlandesa.

Já Vertov, no filme O homem com a câmera, através da câmera ativa do "cinema-olho" (kinoglaz) procura apreender no contexto urbano e na contemporaneidade, não só as atividades do novo homem e mulher soviéticos na cidade, como também evidenciar o próprio processo de 
realização cinematográfica e o que virá a ser um dos primeiros metafilmes da história do cinema.

Enquanto Flaherty se interessa, sobretudo, pela descrição das atividades humanas no contexto em que ocorrem, o que poderá considerar-se um dos pioneiros do cinema antropológico e etnográfico, Vertov vem com seu método experimental desenvolver uma outra perspectiva cinematográfica.

Em relação ao método filmico, é igualmente importante sublinhar o trabalho de Gregory Bateson e Margaret Mead (1942), os quais introduziram nos EUA, o método visual com a utilização da imagem estática (fotografia) e em movimento (cinema) com objetivo de pesquisa. Estes dois pesquisadores, não somente foram precursores na introdução do método fotográfico e filmico na pesquisa, como também nos estudos culturais e comparativos sobre a infância, estudando a educação e a socialização da criança em diferentes grupos e contextos culturais. Só nos anos 1930 em Bali, Nova Guiné e EUA, realizaram diversos filmes nestas áreas geográficas e reuniram 25000 fotografias (BATESON e MEAD, 1938a, 1939, 1942, 1954, 1955).

Ao incorporarem desde o inicio dos seus trabalhos a pesquisa fotográfica, e filmica, a interdisciplinaridade e o método comparativo para observar e analisar os comportamentos em diferentes grupos étnicos e culturais, Mead e Bateson estão na origem dos métodos modernos de pesquisa.

Na França, Marcel Griaule $(1938,1957)$ e Jean Rouch (1948, $1957,1961,1968,1972,1979)$ foram igualmente pioneiros na utilização do filme como método de pesquisa etnográfica, sobretudo na análise dos ritos e das atividades rituais, começando a imagem animada a ocupar um espaço importante nos trabalhos etnológicos e antropológicos.

Rouch $(1949,1972)$ interessa-se particularmente pelas cerimônias religiosas e pelos rituais de iniciação praticados pelos Songhay (Niger), colocando simultaneamente em evidência a organização dos processos de aquisição e transmissão dos rituais e os gestos e modalidades de aprendizagem.

Através de uma "câmera participante" (HEUCH, 1962), de uma câmera em movimento e do que denominou "cinema-verdade", Rouch foi filmar o Homem nas suas atividades banais ou rituais, foi ao encontro do eu e do outro no território africano ou no espaço urbano e familiar na região de Paris. 
Seguiram-se outros pesquisadores da universidade de Paris XNanterre e da universidade de Paris $V$ que têm utilizado o filme no estudo das técnicas do corpo, dos processos de aprendizagem e das atividades educativas e rituais. (FRANCE, 1998; COMOLLI, 1983, 1995; GUERONNET, 1977; LOURDOU, 1975; STORK, 1982,1986).

Claudine de France (1998) desenvolveu as bases conceituais da uma nova disciplina, a antropologia filmica, em seu livro Cinema e Antropologia, constituindo um marco decisivo para o desenvolvimento deste novo domínio cientifico.

Por seu lado, Annie Comolli elaborou uma cinematografia das aprendizagens através dos seus trabalhos relativos à aprendizagem de técnicas materiais, corporais e rituais.

\section{Cinema documentário e antropologia fílmica}

A realização de um filme de pesquisa baseia-se em opções metodológicas, estratégias filmicas precisas e procedimentos éticos. Ao decidir utilizar a câmera como instrumento de pesquisa, o pesquisador deve refletir sobre as possibilidades técnicas de que dispõe e nas questões éticas que se colocam entre o objeto a ser estudado e o método a ser utilizado.

No filme de pesquisa de caráter etnográfico e antropológico e no método que utilizamos o pesquisador é participante e a câmera esta nas mãos do investigador que a movimenta e a torna ativa e participante. Jean Rouch observa que

Hoje todos os operadores do cinema direto sabem movimentarse com a câmera que se tornou a "câmera viva", "cinema-olho" de Vertov. No domínio do filme etnográfico, esta técnica é particularmente eficaz, pois permite uma adaptação à ação em função do espaço, permite penetrar na realidade em vez de deixála desenrolar-se perante o observador (...) para mim, a única maneira de filmar é de caminhar com a câmera, de conduzi-la onde ela é mais eficaz e, de improvisar um outro tipo de "ballet", onde a câmera se torne tão viva quanto os Homens que ela filma. $(1979: 62-63)$ 
Trata-se da utilização de uma câmera implicada e orientada às questões de pesquisa e à observação participante facilitando a inserção no terreno e a familiarização do objeto e sujeitos de estudo, com o pesquisador e com a própria câmera.

Uma fase inicial da pesquisa consiste na inserção do pesquisador junto ao grupo com o qual se deseja trabalhar. É necessário obter a autorização dos participantes para o desenrolar da pesquisa filmica, fase fundamental para a continuidade do trabalho.

É importante respeitar o desenvolvimento natural das atividades, sem a intervenção do pesquisador-cineasta, o que vem fortalecer a relação entre o filmador e o fimado. Quanto melhor for a inserção do pesquisador no campo, mais as pessoas observadas participam no processo e aumenta a relação de confiança e de aceitação que se estabelece entre todos os participantes.

O hábito de o pesquisador se apresentar no local de estudo com os instrumentos filmicos e fotográficos, entra como uma atividade de rotina, familiariza o outro com o pesquisador e seus instrumentos e atenua nas pessoas filmadas a "consciência da câmera" (BATESON, 1942) e os efeitos da "profilmia" (FRANCE, 1998). Os efeitos da câmera, da profilmia, estão sempre mais ou menos presentes durante toda pesquisa filmica, mas eles poderão ser atenuados se observarmos os princípios acima salientados.

Quando filmamos em contextos culturais e meios sociais diferentes daqueles do pesquisador, é necessário combater os estereótipos e as idéias pré-concebidas e desenvolver uma atitude de autocrítica. É importante respeitar os hábitos e costumes das populações que estudamos e não transpor os modelos de nossa própria cultura (BASTIDE, 1958; RAMOS, 1993, 2001; SERAFIM, 2002, 2004)

Ao passarmos da fase de inserção e da observação direta para a observação instrumentalizada, ou seja, com o uso da câmera, o pesquisador é obrigado a elabora uma "reconversão do olhar", ou melhor,

essa reconversão começa com a tomada de consciência, por parte do aprendiz - cineasta, dos traços que permitem distinguir a observação filmica da observação direta. Ela prossegue no difícil aprendizado de uma nova ordem de relações entre os 
diferentes meios de apreensão sensorial. (ROSENFELD, 2000: 51).

Nesta fase do processo de pesquisa, torna-se indispensável a adequação entre a mise en scène do cineasta e a auot- mise en scène das pessoas filmadas. Para Claudine de France

Pelo simples fato de que aceitem ser filmadas, as pessoas observadas se colocam em cena e são testemunhas da intervenção do cineasta. Mise en scène própria às pessoas filmadas $\mathrm{e}$ intervenção do observador-cineasta se manifestam em diversos niveis, mais frequentemente à revelia de seus próprios autores. (...) a observação do etnólogo-cineasta, mesmo a mais distante é sempre "participante". Ou seja, o etnólogo-cineasta participa sempre de alguma maneira, do processo observado, porque sua intervenção e a auto-mise en scène própria das pessoas filmadas são inevitáveis. Reciprocamente, as pessoas filmadas participam do processo de observação porque intervêm da mise en scène do cineasta. (1998: 21-22)

Esta adequação processa-se através da variação de postos de observação, enquadramentos, ângulos de visão, distâncias focais, planos fixos e em movimento, em função das atividades, relações e gestual idades que se pretendam observar e destacar.

Torna-se, desta forma, necessário adotar estratégias diferentes, a fim de resolver os problemas que surgem nas situações particulares, como exemplo podemos citar, entre outros, a situação em que filmamos em espaços exiguos, domésticos atividades relacionadas ao banho, toalete, alimentação e atividades lúdicas (RAMOS, 1993, 2002; SERAFIM, 1994) ou em espaços escolares, como creches, jardins da infância e escolas, onde encontramos um número elevado de participantes e múltiplas atividades ocorrendo simultaneamente (RAMOS 1994, 1996, 1997, 1998, 2000; RAMOS e SERAFIM 2001a, 2001b).

A devolução das imagens aos participantes, ou seja, o feed-back com a discussão e partilha do produto realizado com as pessoas filmadas, permite que estas tenham um controle do material registrado, clarifiquem ou acrescentem elementos que não são claros para o 
pesquisador e, por vezes, para os próprios participantes e introduzem novas relações de pesquisa tornando o filme fruto uma cooperação entre o pesquisador e os sujeitos filmado estabelecendo-se uma relação de intersubjetividade.

A construção final do filme processa-se no momento da edição do material filmado, após múltiplos visionamentos e análise rigorosa e minuciosa do material bruto. O resultado final é, em geral, um documento sóbrio, desprovido de efeitos técnicos (fade, fusão, slow motion etc.) e de comentários orais. O auxilio de um narrador da voz over e voz off só são utilizados nas situações estritamente necessárias do ponto de vista narrativo. Busca-se assim preservar ao máximo a fidelidade ao processo observado, respeitando a cronologia dos eventos e o ambiente natural onde os mesmos ocorrem.

Os produtos resultantes do material coletado e analisado podem ser de vários tipos, Poderão ser, por exemplo, documentos de caráter descritivo ou comparativo. $\mathrm{O}$ documento descritivo aprofundado, desenvolveu-se principalmente a partir dos trabalhos filmicos de Robert Flaherty. Já o documento de tipo comparativo foi iniciado por Gregory

170 Bateson e Margaret Mead, onde se destaca sobretudo o filme Bathing Babies in Three Cultures (1954).

Posteriormente outros pesquisadores desenvolveram esta metodologia realizando, tanto ao nível descritivo como comparativo, diversos documentos filmicos. Ramos (1993, 1995a, 2001, 2002a), Stork, Ramos et al. (1994,1995), Ramos e Serafim (2001a, 2001b, $2001 \mathrm{c}$ ) realizaram diversos filmes comparativos sobre os cuidados às crianças em diferentes culturas, favorecendo a comparação das observações e permitindo salientar as semelhanças, mas também as diferenças dos comportamentos e das atividades humanas.

Outros documentos buscam uma descrição mais aprofundada dos fenômenos estudados a fïm de uma maior compreensão de um determinado grupo ou sociedade. Nesta linha, podemos destacar os trabalhos de Ramos (1994b, 1995b, 1996c, 1997, 1998, 2000a, 2001), de Ramos e Serafim (2001a), realizados em famílias, creches e jardins de infầncia em diferentes paises europeus (Portugal, França, Itália e Romênia) e da América Latina (Brasil).

Nos filmes As mãos que embalam (1999a) e Bercements Tsiganes (1999b) podemos observar em várias famílias de origem cigana, originárias da ex-Yugoslávia e vivendo na Itália, as práticas 
educativas, cuidados às crianças e estilos comunicacionais, sendo evidente como todo o grupo se ocupa da criança, o estilo proximal de interação e a iniciação precoce das crianças nas atividaddees de cuidados maternos.

Já nos filmes Isabelle (Ramos, 1996a), Une famille portugaise à Paris (Ramos, 1996c) e Isthar e Sotis (Ramos, 2000a), podemos observar os estilos educativos e comunicacionais e as técnicas de maternagem, em familias de origem africana e portuguesa, imigrantes respectivamente em França e Itália. Podemos igualmente constatar particularidades relacionadas com a situação de imigração e aculturação, nomeadamente, influências da cultura de origem e da cultura de acolhimento nesses estilos e práticas.

Nos filmes Apaiser le bébé à la créche (Ramos, 1994a), Autour des gestes de maternage (Ramos, 1996b) e Le jour se lève (Ramos, 1997), podemos observar em várias creches e jardins de infância da região centro de Portugal, a dinâmica educativa, os cuidados e estilos interativos das educadoras com as crianças, as diferentes atividades de estimulação e, ainda, como as crianças se exercitam, desde muito cedo, na aprendizagem das técnicas de cuidados maternos.

No filme Grands parents et Petits Enfants (Ramos, 1995a), dedicado à participação dos avós nos cuidados e educação dos netos, podemos observar várias avós e avôs portugueses em atividades de cuidados, tais como, adormecimento, banho e toalete, alimentação, interações lúdicas e de estimulação de bebês no primeiro ano de vida. Observamos, igualmente, uma riqueza e diversidade de estimulações verbais, fisicas e sinestésicas na interação com a criança e práticas religiosas e mágico-religiosas de proteção.

Nesta perspectiva situam-se igualmente alguns trabalhos de José Francisco Serafim (2000a, 2000b, 2000c, 2000d, 2000e, 2000f) realizados junto ao grupo Wasusu sobre os aprendizados a vida quotidiana neste grupo indigena que vive em uma reserva no estado de Mato Grosso.

Serafim realizou com este grupo étnico uma pesquisa filmica aprofundada, tendo realizado vários filmes dedicados ao processo de educação e socialização da criança das crianças e das atividades quotidianas realizadas pelos Wasusu. Os filmes mostram como se processa a socialização da criança, sobretudo, no grupo doméstico e como as aprendizagens ocorrem principalmente por observação, 
imitação e participação. As crianças indígenas iniciam-se muito precocemente nas atividades domésticas, no transporte dos bebês e nos variados cuidados infantis, sendo o modo de comunicação predominante com a criança o contato corporal e visual. Observa-se, por exemplo, através dos documentos filmicos que o contato físico é quase constante em todas as atividades e que a comunicação verbal bastante reduzida.

Constata-se, então, que a metodologia, através da utilização da imagem em movimento fornece uma contribuição valiosa para o desenvolvimento do conhecimento tanto ao nível da pesquisa como da formação. Ela nos dá a descrição tanto das palavras como das posturas e dos gestos, permitindo-nos aceder aos conteúdos verbais e não-verbais. A este propósito Annie Comolli observa em seu texto "Éléments de méthode en anthropologie filmique" que

Descrever através do filme consiste em apresentar de forma aprofundada ou simplesmente fragmentada uma pessoa, um grupo humano, uma atividade ou um conjunto de atividades, um lugar, um momento etc. Trata-se para o cineasta, de explorar, mais ou menos detalhadamente os aspectos sensíveis do objeto de estudo. A descrição filmica diz respeito somente indiretamente ao sensível não acessível pelo filme, como igualmente ao não sensivel. O táctil, o olfativo, o gustativo são simplesmente sugeridos ou indicados pelo filme graças à apresentação de manifestações concretas apreendidas pela vista e pela audição (2003: 16).

\section{Considerações finais}

O filme é um meio de comunicação como outro qualquer e um meio de comunicação intercultural por excelência. São inúmeras as vantagens da utilização da imagem e das técnicas audiovisuais no domínio social, comunicacional, intercultural, psicológico e educacional.

O documento filmico promove uma atitude de descentração (Piaget, 1970) a qual contribui para flexibilizar e relativizar princípios apresentados como únicos e universais, buscando-se evitar comportamentos de intolerância e vindo a facilitar e aprofundar a 
comunicação junto dos diferentes grupos e comunidades (RAMOS, 2003d).

O filme documentário constitui-se em um excelente suporte tanto para a pesquisa fundamental ou aplicada como a formação de diferentes áreas disciplinares.

Claudine de France observa, neste sentido, que:

Tudo nos leva a concluir que os numerosos problemas levantados durante a elaboração de um filme, as reflexões $\mathrm{c}$ as descobertas que esta experiência suscita fazem do cinema etnográfico algo mais que a simples ocasião de armazenar imagens sonoras que virão ilustrar ou completar trabalhos escritos, e do cineasta um pesquisador completo, cuja empreitada engendra progressivamente uma disciplina autônoma que propusemos chamar provisoriamente de antropologia filmica. (1998:401)

É fundamental que a metodologia filmica seja integrada nas diversas ciências sociais e humanas, de modo a favorecer o conhecimento do Homem e das suas atividades nos diferentes contextos e culturas, vindo a possibilitar o estudo do "fato social total" e a promover a análise e a comparação inter/transcultural.

\section{Referências Bibliográficas}

BATESON, G.; MEAD, M. Balinese character, a photographic analysis. New York: The New York Academy of Sciences, 1942.

BATESON, G. et al. . La nouvelle communication. Paris : Seuil, 1981. 1988.

BATESON, G. ; RUESH, J. Communication et Société. Paris : Seuil,

COMOLLI, A. Les gestes du savoir. La Garenne-Colombes : Publidix, 1983.

COMOLLI, A. Cinématographies des apprentissages. Fondements et stratćgies. Paris : Arguments, 1995.

COMOLLI, A. Éléments de méthode em anthropologie filmique. Travaux en Anthropologie filmiquc. Nanterre: Publidix Université Paris $\mathrm{X}$ - FRC, 2003, pp. 5-43.

FRANCE, C. de (ed). Pour une anthropologie visuelle. La Haye : Mouton Ed. et EHESS, 1975. 
FRANCE, C. de . Cinema e antropologia. Campinas : Editora da Unicamp Papiprus, 1998.

ROSENFELD, J. M. Filmar uma reconversão do olhar. FRANCE, C. de (org.) Do filme etnográfico à antropologia filmica. Campinas : Editora da Unicamp, 2000, pp. 43-53.

FRANCE, $X$. de. Eléments de scénographie du cinéma. Nanterre : Univ. de Paris X, 1989.

GRIAULE, M. Masques dogon. Paris: Mémoires de l'Institut d'Ethnologie, XI, 1938.

GRIAULE, M. Jeux Dogon. Paris : Institut d'Ethnologie, 1938.

GRIAULE, M. Méthode de l'ethnologue. Paris: PUF, 1957.

HEUCH, L. Cinéma et sciences sociales. Paris : UNESCO, 1962.

LEROI-GOURHAN, A. Cinema et sciences humaines. Le film ethnographique existe-t-il ? Revue de géographie humaine et d'ethnologie. 3, pp. 42-51, 1948.

LEROI-GOURHAN, A. Le geste et la parole. I. Technique et langage. Paris : A. Michel, 1964.

LEROI-GOURHAN, A. Le geste et la parole. II. La mémoire et les rythmes. Paris : A. Michel, 1965.

MAUSS, M. Les techniques du corps. Journal de Psychologie: (32, 174 3-4), 1934, pp. 271-293.

MEAD, M. The primitive child. In C. Murchison (ed) A Handbook of Child Psychology. Worcester, Mass: Clark University, 1931.

MEAD, M. Une éducation en Nouvelle-Guinée. Paris : Payot, 1973.

MEAD, M.; MacGregor, F. Growth and culture. A photographic study of balinese childhood. New York : G.P.Putman's Sons, 1951.

MEAD, M. Research on primitive children. In L. Carmichel (ed).Manual of child psychology. New York: Wiley, 1954.

MEAD, M. L'anthropologie visuelle dans une discipline verbale. In Pour une Anthropologie visuelle. C.de France (dir.). La Haye : Mouton, 1979.

PIAGET, J. Épistemologie des Sciences de l'Homme. Paris : Gallimard, 1970.

RAMOS, N. Educação precoce e práticas de cuidados infantis em meio urbano. Actas do Colóquio Viver na Cidade. Lisboa: LNEC, 1990, pp. 315-323.

RAMOS, N. Maternage en milieu portugais autochtone et immigré. De la tradition à la modernité. Une étude ethnopsychologique. Tese de Doutoramento em Psicologia. Paris V : Université René Descartes, Sorbonne, 2 vol, 1993a.

RAMOS, N. Le monde enchanté de l'endormissement et de l'apaisement au Portugal. In Les rituels du coucher de l'enfant. Variations culturelles. Paris : E.S.F., 1993b, pp.235-268. 
RAMOS, N. (Org.) Educação intercultural. Mestrado em "Relações Interculturais". Lisboa, Univ. Aberta, 2 vol, 1995.

RAMOS, N. (Org.). Comunicação Intercultural. Mestrado $\mathrm{cm}$ "Relações Interculturais". Lisboa: Univ. Aberta, 2 vol, 2001 a.

RAMOS, N. Comunicação, Cultura e Interculturalidade : Para uma Comunicação Intercultural. Revista Portuguesa de Pedagogia. (35, 2), 2001b, pp.155-178.

RAMOS, N. Educação, saúde e culturas - Novas perspectivas de investigação e intervenção na infância. Revista Portuguesa de Pedagogia. $(1,2,3,36), 2002$, pp. 463-487.

RAMOS, N. Etnoteorias do desenvolvimento e educação da criança. Una perspectiva intercultural e preventiva. Psicologia, Sociedade \& BemEstar. Leiria: Ed. Diferença, 2003a, pp. 161-177.

RAMOS, N. Interculturalité, Communication et éducation. Bucareste: Milena Press. 2003b.

RAMOS, N. O contributo da metodologia filmica para o cstudo das representações sociais: perspectivas de pesquisa na infância. Actas do $5^{\circ}$ Encontro de estudos e pesquisas interdisciplinares em representações sociais. Natal: Univ do Rio Grande do Norte, 2003c, pp. 343-347.

RAMOS, N. Perspectivas metodológicas em investagação: o contributo do método filmico. Revista Portuguesa de Pedagogia, $n^{\circ} 3$, Lisboa, 2003d, pp. 35-62. 2004

RAMOS, N. Psicologia Clinica e da Saúde. Lisboa: Univ. Aberta,

RAMOS, N. A família nos cuidados à criança e na socialização precoce $\mathrm{cm}$ Portugal e no Brasil: uma abordagem intercultural comparativa. Desafios da comparação. Família, mulher e género em Portugal e no Brasil. Oeiras: Celta, 2004, pp.149-190.

REGNAULT, F.L. Les attitudes du corps dans les races humaines. Revue Encyclopédique, 1896, pp. 9-12.

ROUCH, J. Le film ethnographique. In J. Poirier (ed), Ethnologie générale. Paris: Gallimard, 1968.

ROUCH, J. La caméra et les hommes. In C. de France (Ed) Pour une anthropologie visuelle. La Haye: Mouton Éditeur, EHESS, 1975.pp. 5371.

SERAFIM, J. F. Apprentissages de l'enfant et vie quotidienne chez les Wasusu (Mato Grosso, Brésil). Une étude d'anthropologie filmique. Villeneuve d'Ascq : Presses Universitaires du Septentrion, 2002

SERAFIM, J. F. "Antropologia filmica e pesquisa em Ciências Sociais", In Cadernos do GIPE-CIT 11, Salvador, Programa de PósGraduação em Artes Cênicas, 2004, pp. 89-97.

STORK, H. Enfances indiennes. Étude de psychologie transculturelle et comparée du jeune enfant. Paris: Le Centurion, 1986. 
STORK, H. (Dir), Les rituels du coucher de l' enfant. Variations culturelles. Paris : E.S.F, 1993.

\section{Referências Filmográficas}

BATESON, G.; MEAD, M. Childhood rivalyry in Bali and in New Guinea. $35 \mathrm{~mm}$, pb, 15 min., 1938a.

BATESON, G.; MEAD, M. (1938). First days. in the life of a New Guinea baby. $35 \mathrm{~mm}$, pb, $14 \mathrm{~min} ., 1938 \mathrm{~b}$.

BATESON, G.; MEAD, M. Learning to dance in Bali. $35 \mathrm{~mm}, \mathrm{pb}$, 20 min., 1939a.

BATESON, G.; MEAD, M. Karba's first years. $35 \mathrm{~mm}$, pb, $20 \mathrm{~min}$, 1939b.

BATESON, G.; MEAD, M. Bathing babies in three cultures. $35 \mathrm{~mm}$, pb, 10 min., 1954.

COMOLLI, A. Initiation aux techniques domestiques. U-matic, pb, 40 min., 1972.

COMOLLI, A. Initiation aus soins corporels. VHS, pb, $20 \mathrm{~min}$., 1973a.

COMOLLI, A. La petite ménagère. $16 \mathrm{~mm}, \mathrm{c}, 30 \mathrm{~min} ., 1973 \mathrm{~b}$.

176 COMOLLI, A., France, C.de. Techniques de musculation: la leçon. $16 \mathrm{~mm}$, c. $40 \mathrm{~min} ., 1973 \mathrm{c}$.

COMOLLI, A. La toilette. $16 \mathrm{~mm}$, c., 20 min., 1974

COMOLLI, A. Le Sabbat des enfants. U-Matic, c, 20 min., 1983-84.

FLAHERTY, R. Nanook of the North, $35 \mathrm{~mm}$, pb, 55 min., 1922.

FLAHERTY, R. Man of Aran, $35 \mathrm{~mm}, \mathrm{pb}, 75 \mathrm{~min} ., 1934$.

FRANCE, C.de. La Charpaigne. $16 \mathrm{~mm}$, pb, $30 \mathrm{~min} ., 1968$.

FRANCE, C. de. Laveuses. 16mm, c, 30 min., 1970.

GRIAULE, M. Au pays des dogons. $16 \mathrm{~mm}$, pb, $13 \mathrm{~min}$., 1938a.

GRIAULE, M. Sous les masques noirs. $16 \mathrm{~mm}, \mathrm{pb}, 12 \mathrm{~min} ., 1938 \mathrm{~b}$.

GUERONNET, J. Les trois bains. U-Matic, c, 60 min., 1977 a.

GUERONNET, J. Le bain d'Atuyo. Super $8 \mathrm{~mm}, \mathrm{c}, 17 \mathrm{~min} ., 1977 \mathrm{~b}$.

LOURDOU, Ph. (Kebo. Super $8 \mathrm{~mm}, \mathrm{c}, 15 \mathrm{~min} ., 1975$.

LOURDOU, Ph. La vaisselle. Super 8 mm, c, 15 min., 1980.

LUMIERE, L. Le goûter de bebé. $35 \mathrm{~mm}$, pb, 2 min., 1895.

RAMOS, N. Bercements et berceuses en milieu portugais. U-Matic, c., 30 min., 1993.

RAMOS, N. Apaiser et endormir le bébé à la crèche. Betacam SP, c., 25 min., 1994a.

RAMOS, N. Gestes de mères, gestes de pères. Betacam SP, c., 35 $\min ., 1994 b$. 
RAMOS, N. Grands-parents et petits-enfants. Le renouveau du Printemps. Betacam SP, c, 45 min., 1995a.

RAMOS, N. Maternage Portugais. Betacam SP, c., 35 min., 1995 b.

RAMOS, N. Isabelle. Betacam SP, c, 23 min, 1996a.

RAMOS, N. Autour des gestes de maternage. Betacam SP, c, 25 min., $1996 b$.

RAMOS, N. Une famille portugaise à Paris. Betacam SP, c, 20 min., $1996 \mathrm{c}$.

RAMOS, N. Le jour se lève. De la découverte aux apprentissages. Betacam SP, c, 24 min., 1997.

RAMOS, N. Aprender no plural. A escola de todas as cores. (Apprendre au pluriel. L'école de toutes les couleurs. Betacam SP, c, 25 min, 1998, Versão Portuguesa. e Francesa

RAMOS, N. As mãos que embalam. Ciganos em Florença. Betacam SP, c., 14 min., 1999a.

RAMOS, N. Bercements tziganes. Betacam SP, c., 12 mn., $1999 \mathrm{~b}$.

RAMOS, N. Ishtar et Sotis. Premiers liens, premières découvertes. Betacam SP, c, 13 min., 2000a.

RAMOS, N. Primeiras experiências. Primeiras aprendizagens na creche. Betacam SP, c., 18 min., 2000b.

RAMOS, N. Acalantos. Gestos e ritmos de embalar em Portugal e no Brasil. Betacam SP, c, 34 min., 2001.

RAMOS, N. Pais e Filhos. As teias que o amor tece. Betacam SP, c, 33 min., 2002.

RAMOS, N. Frères et soeurs. Betacam SP, c, 50 min., 2002.

RAMOS, N.; SERAFIM, J. F. O jardim de infância. Arco-íris de aprendizagens e culturas. Betacam SP, c., 36 min., $2001 \mathrm{a}$.

RAMOS, N.; SERAFIM, J. F. O despertar da criança através da música na creche. Abordagem intercultural. Betacam SP, c, 33 min., 2001 b.

Ramos, N.; SERAFIM, J. F. Gestos de maternagem no Brasil. Betacam SP, c., 25 min., 2001c.

ROUCH, J. Initiation à la danse des possédés. $16 \mathrm{~mm}, \mathrm{pb}, 25 \mathrm{~min}$, 1947.

ROUCH, J. Moi, un noir. $16 \mathrm{~mm}$, c., $80 \mathrm{~min} ., 1957$

ROUCH, J., Morin, E. Chronique d'un été. $16 \mathrm{~mm}$, pb, 90 min., 1960.

ROUCH, J. Architects Ayorou. 16 min., c, 30 min., 1971.

ROUCH, J. Horendi. 16 mm, c, 90 min., 1972.

ROUCH, J. Initiation. $16 \mathrm{~mm}, \mathrm{c}, 45 \mathrm{~min} ., 1975$.

SERAFIM, J. F. Natacha. Super 8 mm, c, 34 mn, 1994.

SERAFIM, J, F. Kayatisu, le maïs, video, $40 \mathrm{~min}, 2000 \mathrm{a}$.

SERAFIM, J. F. Musique de flûtes, vídeo, $40 \mathrm{~min}, 2000 \mathrm{~b}$. 
SERAFIM, J. F. La pêche à la nivrée-Husinousu, vídeo, $29 \mathrm{~min}$, $2000 \mathrm{c}$.

SERAFIM, J. F. Le singe et le pécari, vídeo, $43 \mathrm{~min}, 2000 \mathrm{~d}$.

SERAFIM, J. F. Les bains de Sandri. Super $8 \mathrm{~mm}, 25 \mathrm{~min}, 2000 \mathrm{e}$.

SERAFIM, J. F. . Flûtes sacrées. Super $8 \mathrm{~mm}$, c. $25 \mathrm{~min}, 2000 \mathrm{f}$.

STORK, H. Pour endormir Lakshmi. 16 mm, 19 min, 1982a.

STORK, H. Seliamedu. Petits soins aux bébés dans un village Tamoul. $16 \mathrm{~mm}, \mathrm{c}, 32 \mathrm{mn}$., $1982 \mathrm{~b}$.

STORK, H. et al. Techniques de maternage dans différentes cultures. Umatic, c, 52 min., 1988.

STORK; H.; Ramos; N.et al. Bercements et berceuses dans différentes cultures. Betacam SP, c, 24 min., 1994.

STORK, H.; Ramos, N.et al. Le rituel du bain à travers les cultures (Afrique, Asie, Europe).Betacam SP, c, 54 min., 1999.

VERTOV, D. O homem com a câmera. $35 \mathrm{~mm}$, pb, $95 \mathrm{~min} ., 1929$. 